Résumés des conférences et travaux

\title{
Sources et histoire de la tradition sanskrite
}

\section{Sources et histoire de la tradition sanskrite}

Conférences de l'année 2014-2015

Jan E. M. Houben

\section{(2) OpenEdition \\ Journals}

\section{Édition électronique}

URL : https://journals.openedition.org/ashp/1876

DOI : 10.4000/ashp.1876

ISSN : 1969-6310

\section{Éditeur}

Publications de l'École Pratique des Hautes Études

\section{Édition imprimée}

Date de publication : 1 septembre 2016

Pagination : 292-297

ISSN : 0766-0677

Référence électronique

Jan E. M. Houben, "Sources et histoire de la tradition sanskrite », Annuaire de l'École pratique des hautes études (EPHE), Section des sciences historiques et philologiques [En ligne], 147 | 2016, mis en ligne le 05 octobre 2016, consulté le 06 juillet 2021. URL : http://journals.openedition.org/ashp/1876 ; DOI : https://doi.org/10.4000/ashp.1876 


\title{
SOURCES ET HISTOIRE DE LA TRADITION SANSKRITE
}

\author{
Directeur d'études : M. Jan E. M. HoubeN
}

Programme de l'année 2014-2015 : I. Introduction au sanskrit, lingua franca philosophique et scientifique (cours de master). - II. La maison védique et son monde. - III. Pāṇini sans détour : thèmes grammaticaux, problèmes linguistiques, domaines de realia.

I. Dans les plus anciennes réflexions sur le sanskrit, la langue est conceptualisée comme daivi $v \bar{a} k$, une langue sacrée ou divine. Pourtant, dans la pratique, le sanskrit a fonctionné au travers des siècles et des millénaires comme une lingua franca, surtout quand, vers le milieu du premier millénaire, les bouddhistes et les jainas commencèrent à utiliser le sanskrit pour les discussions, les polémiques et les traités religieux, philosophiques et scientifiques, et à conceptualiser le sanskrit dans cette perspective. Les deux approches opposées sont représentées dans les dernières kārikās du livre I du Vākyapadīya de Bhartronari ( $c a 450$ à 510 notre ère). Le sanskrit est la première et, pour très longtemps, la seule langue dans le monde accessible au moyen de grammaires fiables (Pāṇini, $\mathrm{IV}^{\mathrm{e}}$ siècle avant notre ère, Candra, $\mathrm{V}^{\mathrm{e}}$ siècle) et relativement faciles (Kaumāralāta et Kātantra, IV ${ }^{\mathrm{e}}$ - début $\mathrm{V}^{\mathrm{e}}$ ), et son étude devient une entreprise faisable et utile. Après le sanskrit, la langue qui devient ainsi accessible est l'arabe, par la grammaire de Sibawayhi $\left(\mathrm{VIII}^{\mathrm{e}}\right)$, originaire d'une région quelque part entre la Perse et l'Inde, comme Pāṇini plus qu'un millénaire avant lui. (L'usage des anciens textes grammaticaux du prakrit et du tamoul reste très limité.) Même avec l'énorme expansion sociolinguistique du sanskrit depuis au moins le $\mathrm{IV}^{\mathrm{e}}$, les milieux traditionnels continuent à souligner que l'étude du sanskrit, sa grammaire et prosodie, est un devoir rituel. Un texte relativement peu étudié (et non traduit), le commentaire mrtasamjīvinī « ranimant ce qui est mort» de Halāyudha (date estimée : $\mathrm{x}^{\mathrm{e}}$ siècle), l'a bien exprimé par rapport à la prosodie (Chandas-Śāstra de Pingala, édition par Pdt. Kedāranātha, Bombay 1927, p. 2) :

iha hi traivarnikīnāì sāngavedādhyanam āmnāyate / arthāvabodhaparyantaś cādhyayanavidhih / vedāingam ca chanda / tatas tadadhyayanavidhitvāt tad anuștheyam / atha 'triștubhā yajati, brhatyā gāyati, gāyatryā stauti' ity evamādiśravanāt arthāyātam anuș̣tubhādijñānam / kim ca chandasām aparijñānāt pratyuta pratyavāyah śrūyate / yathā - 'yo ha vā aviditārșeyacchandodaivatabrāh manena mantrena yājayati vādhyāpayati vā sa sthānum varcchati gartaì vā patati pramīyate vā pāpìyān bhavati yātayāmānny asya chandāmisi bhavanti'/ tasmāc chandojñānam kartavyam tadartham idam śāstram ārabhyate /

Car dans ce monde l'étude du Veda et ses disciplines auxiliaires par les membres des trois premiers états est mentionnée dans les textes sacrés. Et la prescription d'étudier (est valable) jusqu'à la compréhension (de la matière). Et la prosodie (chandas) est une discipline auxiliaire (i.e., une de ces disciplines auxiliaires obligatoires). C'est pourquoi 
ceci doit être accompli, à cause de la prescription d'étudier la prosodie. Ensuite, parce que les textes révélés disent des choses telle que « il fait l'adoration rituelle par le mètre Trișțubh, il chante par le mètre Brọhatī, il loue par le mètre Gāyatrī », la connaissance du mètre Anușțubh, etc. devient un objectif. Par contre, une connaissance incomplète des mètres entraîne un résultat négatif. Par exemple : "Celui qui exerce une fonction rituelle dans un sacrifice ou qui enseigne par un mantra dont il ne connait pas le voyant, le mètre, la divinité et l'explication traditionnelle, il se heurte à un tronc d'arbre, ou bien il tombe dans une fosse, ou bien il périt (:) il devient pire, ses mètres deviennent délabrés. »C'est pourquoi la prosodie doit être pratiquée : avec ce but nous commençons cette discipline.

Pourtant ce sont les Prātiśākhyas qui sont spécifiquement consacrés à la métrique védique. Le Chandas-Śāstra de Pingala est plutôt le plus ancien texte entièrement consacré à la prosodie, séculière beaucoup plus que védique. Comme la grammaire de Pāṇini, ce texte ne connaît pas de distinction d'écoles, et s'adresse à tous les intéressés. Comme la grammaire de Pāṇini, mais à une échelle beaucoup plus modeste, le Chandas-Śāstra définit sa propre terminologie technique.

Dans le chapitre I, le Chandas-śāstra renvoie à huit schémas métriques de base trois fois léger ou lourd, donc $2^{3}=8$ possibilités - et attribue à chacun un phonème comme " étiquette ». Pour des raisons mnémotechniques et peut-être éducatives, le commentaire de Halāyudha projette ensuite une discussion entre maître et élève sur cette énumération de schémas et phonèmes. Selon cette discussion et selon des indications internes, la séquence des sūtra peut être reconstruite ; elle reste comme transmise, sauf à la fin :

\begin{tabular}{|c|c|c|}
\hline maître : & [1] dhīḥ śrī strī m & $(\mathrm{m}(\mathrm{a}-)---)$ \\
\hline & [2] varā sā y & $(\mathrm{y}(\mathrm{a}-) \cup--)$ \\
\hline élève & [3] kā guhā r r & $(\mathrm{r}(\mathrm{a}-)-\cup-)$ \\
\hline maître, réponse à [3] : & [4] vasudhā s & $(\mathrm{s}(\mathrm{a}-) \cup \cup-)$ \\
\hline élève : & [5] sā te kva t & $(\mathrm{t}(\mathrm{a}-)--U)$ \\
\hline & [6] kadā sa j & $(j(a-) \cup-\cup)$ \\
\hline & [7] kim vada bh & $(b h(a-)-\cup \cup)$ \\
\hline maître, réponse à [7] : & [8] na hasa n & $(\mathrm{t}(\mathrm{a}-) \cup \cup \cup)$ \\
\hline réponse à [5] : & [9] $\mathbf{g r}_{\Omega} \mathbf{l}$ & $(\cup)$ \\
\hline & [12] he (g) & $(-)$ \\
\hline & $\begin{array}{l}\text { ([10] [g] ante) } \\
{[11] \text { dhrādipar }}\end{array}$ & \\
\hline
\end{tabular}

Dans le cadre de notre étude de la prosodie pour le niveau master et en tant que préparation pour un colloque, nous étudions Antoine Chézy (aussi connu comme AntoineLéonard de Chézy) qui occupa la chaire de sanskrit fondée à Paris en novembre 1814 (discours inaugural le 16 janvier 1815). Sur le continent (khanda) dont le nom «Europe » suggère qu'il devrait avoir une "large vision» (uruprekṣa : ayant une uruprekșā), Chézy était alors celui qui était pour la première fois (prathamatah) nommé professeur (prādhyāpakatve), spécifiquement en tant qu'enseignant (upadeștrr) de la langue sanskrite (samiskrtā vāk). Deux siècles après Chézy, ses idéaux de recherche ont-ils été réalisés ou détournés ou oubliés ? On pourrait examiner ces questions sur 
la base d'une analyse des publications de Chézy et de plusieurs sources qui reflètent la «Renaissance orientale» de son époque. Quelques points principaux. Après avoir étudié de nombreuses strophes de type śloka (ślokasyaughān pațhitvā), Chézy publia sa Théorie du Śloka en 1827, avec comme titre sanskrit : ślokaracanāvidhi. Chézy y introduisit le sujet de la métrique par deux citations de Voltaire (1694-1778, renommé partout en Europe et toujours profondément admiré par Chézy), développa des schémas mnémotechniques pour ceux qui sont familiers avec la métrique occidentale, et résuma sa conclusion en sanskrit, en deux strophes (śloka). Une autre publication importante parut en 1830 : la première édition (śodhita-śāstra) du texte d'une pièce de théâtre composée par le grand poète indien Kālidāsa, le Śākuntala ou Abhijñānaśakuntala (avec traduction française, notes, le récit selon le Mahābhārata en sanskrit et en persan), déjà connu, voire célèbre en Europe à travers une traduction charmante mais encore assez imparfaite en anglais par Sir William Jones (Calcutta 1789; Londres 1790). Sous le nom partiellement latinisé d'Apudy, Chézy publia encore une sélection de 51 strophes de l'Amaruśataka : texte en sanskrit, traduction, notes et gloses, une Anthologie érotique d'Amarou (1831). C'était un an avant qu'il compte, comme son collègue Abel-Rémusat, parmi les 19000 victimes de l'épidémie de choléra à Paris. D'après ce survol rapide, il est clair qu'A.-L. de Chézy, avec un grand enthousiasme durant plusieurs décennies, avait maîtrisé le sanskrit - jusque-là toujours apprécié pour sa fonction expressive et sa fonction d'archive - jusqu'à un niveau rarement égalé par ses contemporains et successeurs, et qu'il avait rendu, dans le contexte de son époque, des services (upakrta) importants et originaux à l'étude du sanskrit et de sa littérature : il s'était montré passionné par le sanskrit et son upakrtin dans le sens de Pāṇini, AA 5.2.88, iștādibhyaś ca (avec iștādigaṇa, ișta ... upakrta ...).

Nous pouvons ensuite honorer Antoine-Léonard de Chézy en composant une stanza (Sragdharā) qui reprend l'essentiel des remarques précédentes :

prādyaut śākuntalātmā yadupakṛititayā śodhite śāstrarūpe

śokasyaughān paṭhitvā racanavidhim akārṣît kavīnām prasiddhyai /

vācah prādhyāpakatve prathamata upadeștābhavat samiskrtāyāh

so’yam "Lévnārd da Chézy" "Pari”-nagara "Uruprekṣa"-khaṇḍe samaste //

II. Dans notre étude de la maison védique et son monde nous continuons d'abord notre analyse du Śrī sūkta, un hymne connu jusqu'aujourd'hui pour des usages « domestiques ». Nous étudions la version de l'Āśvalāyana-samhitā (ĀS) récemment rendue accessible grâce à l'édition de $\mathrm{B}$. B. Chaubey $(\mathrm{BBCh})$ et discutée dans les rapports des années précédentes. Nous comparons le texte avec celui disponible dans le RVkhila. Notre attention est surtout retenue par la stanza 4, dont $\bar{A} S$ donne le texte suivant :

kấm sosmitấm híranyaprākārāam ārdrấm jválantīìn trptấm tarpáyantīm / padmesthitấm padmávarṇām tấm ihópa hvaye śriyam //

kấm sosmitấm (? ainsi BBCh) est problématique, et l'interprétation que BBCh en donne est inacceptable. kấm est interprété comme kấm api, et sosmitấm probablement comme susmitám. La séquence correspond à un passage guère moins problématique dans le RoVkhila, que Scheftelowitz édite comme kāmisy ásmi tấm. Il est d'ailleurs 
impossible d'accepter la proposition de Bhise (Pune 1995) que ce soit une « irregular form of the Des. of kam "to desire, love" " (Whitney note comme thème du désid. : cikamișa). Métriquement nous avons : $1011 / 88$.

a : serait un pāda en Anuștubh avec kāminsî́ au lieu de kấm sosmitấm / kāànsy ásmi tấm, au lieu de la forme d'occurrence singulière (hapax legomenon) kāims comme dérivé de kam «désirer » selon l'analyse de Scheftelowitz, mais qui aurait pourtant dû être kamsì ; si on peut reconstruire un tel mot dans le sens voulu, est-ce qu'un public contemporain aurait pu le comprendre dans ce sens? Le poète aurait pu utiliser, pour exprimer le même sens, une forme qui soit l'équivalent du point de vue du mètre et de l'accent : kāànkșì (nom. de kāinkșa + in MW268.1; les mots en thème $-a+i n$ sont bien connus; exemples de $-\bar{a}+i n$ sont : manissín et śikṣin; dans le dernier, comme, *kāñkșin*, in est affixé à un substantif en -a basé sur un thème verbal du désidératif). kāmisí peut-il être une corruption ou une forme prākritique / hybride, originale ou substituée, de kāànkṣ̂́ ? Ensuite, ásmi, superflu du point de vue syntaxique, sémantique et métrique (comme, d'ailleurs, tấm), peut avoir été ajouté pour l'emphase dans le contexte rituel, à un certain stade de la transmission. Ou bien, c'est une explication ajoutée dans la transmission. En supprimant les ajouts qu'on peut comprendre comme dérivant de la pratique rituelle, on arrive donc à un pāda Anuștubh, *kāinkș̣ ${ }^{*}$ * híranyaprākārām.

Quant à -prāvārām (Scheftelowitz), BBCh donne -prākārām dans le texte mais traduit - prāvārām.

b : sans jválantīim (superflu du point de vue sémantique, arrivé en 4 de 5), on a un deuxième pāda Anușțubh.

Samihitā-pātha reconstruit des pādas ab :

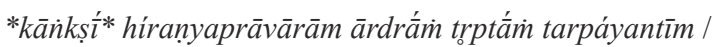

Pada-pāṭha reconstruit :

*kāìkș̣̂́* / [...] híranya ${ }^{\circ}$ prāvārām / ārdrấm / trptấm / tarpáyantīm /

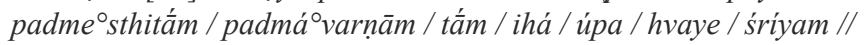

Traduction (de la version reconstruite) :

Désireux, j'appelle ici, près de moi, elle dont le vêtement est d'or, ruisselante / douce, comblée et qui nous comble, qui reste debout dans un lotus, qui a la couleur d'un lotus, Śrī.

Nous étudions ensuite en détail quelques scènes domestiques dans le Śatapatha Brāhmaṇa, notamment l'histoire de Purūravas et Urvaśī.

III. Dans nos efforts pour comprendre Pāṇini dans son propre contexte, il est inévitable que nous l'approchions à travers les textes qui rendent son œuvre grammaticale accessible, les huit livres de règles grammaticales (Aștādhyāyī, AA) et ses annexes, et que nous utilisions l'information disponible sur les conditions historiques de son travail. La primordialité de l'oralité dans le milieu de Pāṇini est évidente, même s'il était familier avec l'écriture, si lipi et libi dans AA 3.2.21 sont acceptés dans le sens 
d'« écriture », et si la formation de yavanānī selon AA 4.1.49 vise, avec Kātyāyna, yavanānī lipih̆, l' « écriture grecque ». Une discussion très détaillée sur l'oralité de Pāṇini et sur la position marginale de l'écriture, même chez les auteurs plus tardifs dans la tradition, se trouve dans l'étude de Madhav M. Deshpande, «From orality to writing: transmission and interpretation of Pāninini's Așțādhyāyī » (dans Veda-Vedānga et Avesta entre oralité et écriture [section IIIA, sous la dir. de J. E. M. Houben et J. Rotaru], Travaux du symposium international - Le Livre, la Roumanie, l'Europe, t. III, p. 57-100, Bucarest, 2011). Pāṇini était donc familier avec l'écriture, mais dans la transmission et la composition des règles de la grammaire par lui, ses prédécesseurs et ses successeurs immédiats, l'écriture n'était pas utile et n'était pas utilisée. Plus tard dans la tradition de l'école de Pāninini, l'écriture devient importante mais la maîtrise de la grammaire sans support écrit reste la base pour son utilisation par les savants.

Pour comprendre l'œuvre grammaticale de Pānini, la recherche de sa structure a été primordiale depuis que sa grammaire a été découverte à la fin du XVIII ${ }^{\mathrm{e}}$ et au début $\mathrm{du} \mathrm{XIX}{ }^{\mathrm{e}}$ siècle. Un diagramme en arbre qui montre le système et les divisions systématiques dans cette grammaire a été présenté dans le rapport de l'année dernière. Des éléments incompatibles ou contrastants dans sa grammaire, cependant, ont suggéré qu'il y avait des sources distinctes pour quelques éléments et sections. Pāninini lui-même mentionne des prédécesseurs, au nombre de dix, mais, dans l'ensemble, il renvoie assez rarement à leur travail dans les quelques 4000 règles que compte sa grammaire. Le débat sur le système de la grammaire de Pāṇini et les incohérences dans le système perçu avait été porté à un nouveau niveau par S. D. Joshi et J. A. F. Roodbergen qui démontrent dans un article, "The structure of the Așțādhyāyī in historical perspective » (dans Proceedings of the International Seminar on Studies in the Aștādhyāya of Pānini, S. D. Joshi et S. D. Laddu [éd.], p. 59-95, Pune, 1983), une rupture méthodologique profonde entre de larges sections de la grammaire de Pāninin. Afin d'expliquer cette rupture ils ont proposé que certaines parties proviennent d'une source post-pāṇinéenne et qu'elles aient été ajoutées à la grammaire lorsque Kātyāyana écrivait ses aphorismes de commentaire. La proposition de Joshi et Roodbergen et les quelques critiques détaillées qu'elle a reçu ont été brièvement discutées par nous en 2003 dans « Three Myths in Modern Pāṇinian Studies », Asiatische Studien - Études asiatiques 57.1, p. 121-179.

Nous étudions plusieurs sūtra ou règles (a) qui concernent la dérivation des pada (normalement : " mot», dans certains conditions, « thème ", comme discuté auparavant), à partir des éléments tels que les dhātu, « racines », pratyaya, " suffixe », en renvoyant au rôle syntaxique du mot visé dans la phrase au travers du schéma des $k \bar{a} r a k a$, «facteurs dans une action », surtout les sūtras qui enseignent les suffixes $k r t$; et (b) des sūtra ou règles qui dérivent des pada à partir des pada déjà dérivés, sans aucun renvoi au schéma des kāraka, surtout les sūtras qui enseignent les suffixes taddhita.

La conclusion émergeant inéluctablement de notre étude est que la première étape dans l'argument de Joshi et Roodbergen est acceptable : la grammaire attribuée par la tradition à Pāṇini est forgée en un système intégré et remarquablement cohérent, mais des sections considérables de cette grammaire ont été soumises de façon différenciée aux efforts de plusieurs auteurs et rédacteurs successifs. Le contexte nous oblige 
ensuite à distinguer, partiellement à la différence de Joshi et Roodbergen, au moins un prédécesseur principal - hors des dix maîtres antérieurs marginalement mentionnés dans le texte - et un successeur ou élève, possiblement Pānini, qui introduisit quelques innovations importantes mais limitées au système hérité.

Nous constatons finalement qu'il y a peu de théories sur l'emploi des données relatives au contexte social des faits linguistiques du passé, y compris sur le contexte social des faits linguistiques rapportés dans la grammaire de Pāninini. Tout comme la linguistique structurale et générative a un précurseur, Ferdinand de Saussure (18571913) - qui a été plusieurs fois explicitement reconnu comme tel, par exemple par Noam Chomsky, la sociolinguistique, et surtout la sociolinguistique historique, a aussi un précurseur important avec Antoine Meillet (1866-1936), pour qui la langue était «éminemment un fait social» (A. Meillet, Linguistique historique et linguistique générale, Paris, 1921, p. 230, ) - mais, hors d'une mention isolée (L.-J. Calvet, $L a$ sociolinguistique, $3^{\mathrm{e}}$ édition, Paris, 1998), il a rarement été reconnu comme tel.

Le 16 avril 2015, Dr Samudrala Venkatabala Gupta donne une conférence, lors de notre cours, sur « Devanāgarī-OCR et la numérisation des œuvres grammaticales en sanskrit ». Le 21 mai 2015, une conférence est donnée dans le cadre de notre cours : Dr D. N. Mishra parle de « Plantes médicinales pour Jvara Cikitsā (traitement de la fièvre) dans les textes āyurvédiques ». Dr Mishra nous expliqua que la connaissance actuelle des plantes médicinales āyurvédiques est dans une large mesure basée sur les pratiques traditionnelles, souvent issues de sources familiales ou de textes médicaux sanskrits, en particulier de la Caraka-Samhitā, Suśruta-Samhitā, AșțāngahṛdayaSamhitā, Siddhasāra, Mādhavanidāna et Mādhavacikitsā, etc. Dans cette littérature, de nombreuses plantes sont mentionnées dans les vers, sous leurs noms sanskrits, pour guérir une maladie, ou pour leur rôle complémentaire dans la composition d'un médicament et le choix d'un traitement. À plusieurs reprises, le nom d'un groupe de plantes est mentionné, représentant généralement deux ou plusieurs plantes individuelles (Triphalā, Pañcamūlī, Bṛhatyādigaṇa, etc.). Des espèces de plantes taxonomiquement entièrement différentes sont entrées en usage sous le même nom de groupe. Nous trouvons aussi dans les textes des listes différentes de plantes ayant un goût amer, astringent, piquant, aigre, doux, pour le même traitement. Par défaut, on comprend dans l'Āyurveda qu'une partie d'une plante signifie la partie souterraine ou « racine », sauf si d'autres parties sont explicitement mentionnées. Les interprétations des textes sanskrits pertinents ont été affinées grâce aux progrès de la connaissance des médicaments bruts et la compréhension de l'emplacement des principes actifs dans certaines parties de la plante. Des problèmes dans l'interprétation textuelle - expressions ambiguës, identifications botaniques divergentes et le manque d'un registre adéquat des données cliniques qui confirment l'efficacité et les résultats de l'utilisation de médicaments à base de plantes - nous obligent à mettre en place des études scientifiques coordonnées afin de corriger ces lacunes. 